\title{
Letter
}

\section{Topological insulation in a ladder model with particle-hole and reflection symmetries}

\author{
B Hetényi ${ }^{1,2,3}$ (D) and $M$ Yahyavi ${ }^{1}$ (1) \\ 1 Department of Physics, Bilkent University, TR-06800 Bilkent, Ankara, Turkey \\ 2 MTA-BME Exotic Quantum Phases 'Momentum' Research Group, Department of Physics, Budapest \\ University of Technology and Economics, H-1111 Budapest, Hungary \\ E-mail: hetenyi@fen.bilkent.edu.tr and hetenyi@bme.phy.hu
}

Received 12 December 2017, revised 25 January 2018

Accepted for publication 2 February 2018

Published 20 February 2018

\begin{abstract}
A two-legged ladder model, one dimensional, exhibiting the parity anomaly is constructed. The model belongs to the $C$ and $C I$ symmetry classes, depending on the parameters, but, due to reflection, it exhibits topological insulation. The model consists of two superimposed Creutz models with onsite potentials. The topological invariants of each Creutz model sum to give the mirror winding number, with winding numbers which are nonzero individually but equal and opposite in the topological phase, and both zero in the trivial phase. We demonstrate the presence of edge states and quantized Hall response in the topological region. Our model exhibits two distinct topological regions, distinguished by the different types of reflection symmetries.
\end{abstract}

Keywords: topological matter, one dimensional systems, band structure

(Some figures may appear in colour only in the online journal)

\section{Introduction}

The analysis of topological insulators (TI) in the light of non-spatial symmetries [1-4] was a very crucial step in our understanding of such systems. Non-spatial symmetries, timereversal (TRS), particle-hole (PHS), and their combination, chiral symmetry (CS) lead to the 'ten-fold way' characterization. Based on whether the TRS or PHS operators square to plus or minus one it is possible to establish a 'periodic table' of TIs, which predicts the topological index (none, $\mathbb{Z}$, or $\mathbb{Z}_{2}$ ) for a system with a given dimensionality. The original KaneMele model $[5,6]$ is a TRS- 1 system (its TRS operator squares to minus one), which exhibits Kramers degeneracies at timereversal invariant points in the Brillouin zone. Recently, the effects of spatial symmetries have also been considered [7-9] in the classification of TIs. The interplay of reflection operators with TRS, PHS, and CS can lead to new topological states even in cases in which the original classification schemes [13 ] indicate a zero topological index, and the 'ten-fold way' has

\footnotetext{
${ }^{3}$ Author to whom any correspondence should be addressed.
}

been extended to include additional topological classes [7, 8]. There are several examples $[9,10]$ of topological insulation as a result of TRS and mirror symmetry. From the extended studies of Chiu et al $[7,8]$ topological behavior should also result from the interplay of PHS and reflection.

Known PHS-1 based topological systems ( $C$ and $C I$ symmetry class) are topological superconductors and they are two [11] or three [12, 13] dimensional. In the absence of spatial symmetries one-dimensional systems exhibit a zero topological index. It is only when reflection is present, and when the reflection operator anti-commutes with the PHS operator that non-trivial topological behavior is expected.

In this paper we construct a $1 \mathrm{D}$ model, which exhibits PHS. Gap closure occurs at finite parameter values separating two different quantum phases. The PHS operator for the model squares to minus one, therefore, the model falls in the $C$ and $C I$ classes $[1,2]$, however, the operator $R$ which inverts the legs of the ladder anticommutes with the PHS. Thus, our model has a $2 M \mathbb{Z}$ topological index according to the classification of Chiu et al [7,8]. We also show that our model can be viewed as two models superimposed, each of which 
individually exhibits nontrivial topological behavior. The submodels are Creutz models $[14,15]$ with an external potential. The topological invariant of the complete system is the mirror winding number. We find edge states and a quantized Hall conductance in the topological phase. Applying the Peierls phase to another set of bonds results in the same topological behavior, but with reflection about a bond midpoint playing the role of $R$. We also consider adding a term which mixes the contributions from the two models but is PHS invariant. The winding number displays the same behavior, the edge state become nondegenerate.

\section{Model}

The model is effectively 1D, inversion and time-reversal symmetries are broken simultaneously. The model is a two-legged ladder model with an on-site potential and diagonal hoppings with a Peierls phase. The hoppings connecting the different legs of the ladder perpendicularly move the positions of the gaps within the reduced Brillouin zone (BZ) towards the origin. Turning on a finite flux along the diagonal bonds allows closure of either gap.

Our model is represented in figure 1. The legs of the ladder are one-dimensional tight-binding models with an alternating on-site potential of strength $\Delta$. We study the model at half-filling. The hopping parameter for hoppings along the legs is $t_{x}, t_{y}$ denotes hoppings perpendicular to the legs, and $t_{x y}$ denotes diagonal hoppings. A Peierls phase of $\Phi$ is introduced along the diagonal bonds. Since the phases are directed such that they close around squares, they can be viewed as magnetic impurities placed along the $t_{y}$ bonds. In contrast to the Haldane model [16] (HM), the fluxes in neighboring closed squares circulate in the opposite direction, corresponding to an antiferromagnetic line of impurities. If $t_{y}$ and $t_{x y}$ are zero, but $t_{x}$ and $\Delta$ are finite, the model exhibits two equal gaps at the edge of the reduced BZ (where the energy difference at half-filling is minimum). Turning on $t_{y}$ moves both of these gaps towards the origin. Turning on $t_{x y}$ and a finite $\Phi$ allows the closing of either gap without closing the other. An external magnetic field applied perpendicular to the ladder is indicated by the other Peierls phase $\Phi_{B}$.

The Hamiltonian of the model in reciprocal space (taking the lattice constant to be unity) can be written as a $4 \times 4$ matrix as

$$
\begin{aligned}
H(\Phi, 0)= & \sum_{k}\left[\left(\begin{array}{cc}
0 & -2 t_{x y} \cos (k) \cos (\Phi) \\
-2 t_{x y} \cos (k) \cos (\Phi) & 0
\end{array}\right) \otimes I_{2}\right. \\
& +\left(\begin{array}{cc}
-2 t_{x} \cos (k) & -t_{y} \\
-t_{y} & -2 t_{x} \cos (k)
\end{array}\right) \otimes \tau_{x} \\
& \left.+\left(\begin{array}{cc}
\Delta & 2 t_{x y} \sin (k) \sin (\Phi) \\
2 t_{x y} \sin (k) \sin (\Phi) & \Delta
\end{array}\right) \otimes \tau_{z}\right] .
\end{aligned}
$$

In equation (2) $I_{2}$ denotes the $2 \times 2$ identity matrix, and $\tau_{x} \tau_{y}, \tau_{z}$ denote the Pauli spin matrices. The overall Hilbert space can be viewed as a direct product of two two-dimensional spaces. The Pauli matrices in equation (2) act in the 'right' subspace. A rather convenient fact is that the matrix

$$
U=\frac{1}{\sqrt{2}}\left[\left(\begin{array}{cc}
1 & 1 \\
1 & -1
\end{array}\right) \otimes I_{2}\right]
$$

will diagonalize all three of the matrices on the left of each of the terms comprising $H(\Phi, 0)$, and the gap closure conditions can be readily obtained. The second term of $H(\Phi, 0)$ results in a diagonal $2 \times 2$ matrix with elementss $\lambda_{x}^{ \pm}=-2 t_{x} \cos (k) \pm t_{y}$ times $\tau_{x}$. Requiring that either one is zero gives the values of $k$ at which the gaps reside $\left(k^{ \pm}\right)$. The third term becomes a diagonal $2 \times 2$ matrix with elements $\lambda_{z}^{ \pm}=\Delta \pm 2 t_{x y} \sin (k) \sin (\Phi)$ multiplying $\tau_{z}$. Substituting either of $k^{ \pm}$and requiring that one of the $\lambda_{z}^{ \pm}$is zero leads to a band structure in the reduced BZ with one gap closed (either at $k^{+}$or at $k^{-}$). Two examples of the band structure as a function of the external parameters are shown in figure 2. The phase diagram is shown in the inset of figure 2. Gap closure occurs at the lines.

The shape of the phase diagram, and more importantly, the meaning of the parameters on the axes $(\Delta$ versus $\Phi)$ bears a definite resemblance to the HM [16]. The main reason for this is that like in the HM, inversion symmetry is broken via an onsite potential, and simultaneously, TRS is broken by introducing a Peierls phase on second nearest neighbor hoppings. If one was to apply Haldane's steps in a one-dimensional chain (Rice-Mele model with Peierls phase on second nearest neighbor bonds), no gap closure would result, since in this case the distance between two nearest neighbors is twice the lattice constant, and the contribution at the gap closure points (which are at the edge of the RBZ) would be zero. The ladder configuration allows for second nearest neighbors whose length is not equal

$$
H\left(\Phi, \Phi_{B}\right)=\sum_{k}\left(\begin{array}{cccc}
\Delta & -2 t_{x} \cos \left(k+\Phi_{B}\right) & -2 t_{x y} \cos (k+\Phi) & -t_{y} \\
-2 t_{x} \cos \left(k+\Phi_{B}\right) & -\Delta & -t_{y} & -2 t_{x y} \cos (k-\Phi) \\
-2 t_{x y} \cos (k+\Phi) & -t_{y} & \Delta & -2 t_{x} \cos \left(k-\Phi_{B}\right) \\
-t_{y} & -2 t_{x y} \cos (k-\Phi) & -2 t_{x} \cos \left(k-\Phi_{B}\right) & -\Delta
\end{array}\right) .
$$

We first focus on the case $\Phi_{B}=0$. In this case it is convenient to write the Hamiltonian as a sum of three terms, each of which is a direct product of a $2 \times 2$ matrix and one of the Pauli matrices as to two lattice constants, and the closing of the gap at either $k^{+}$ or $k^{-}$. We note in passing that a Haldane like phase diagram is exhibited in an extended SSH model, in which the Peierls phases on hoppings between different sublattices differ [17]. 


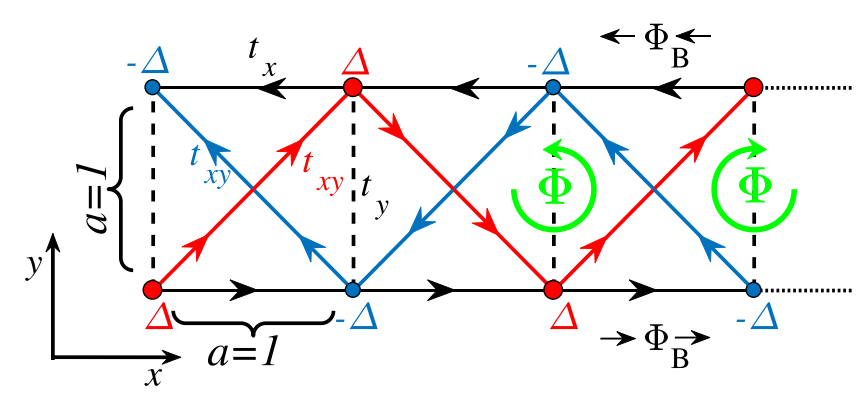

Figure 1. Ladder model. The hopping parameters are defined as follows: $t_{x}$ denotes hopping along the legs of the ladder, $t_{y}$ denote the hoppings perpendicular to legs, $t_{x y}$ denotes hoppings occurring diagonally between legs, connecting second nearest neighbors. The sites in red(blue) indicate where the site depedendent potential is positive(negative). A Peierls phase of $\Phi$ is introduced along the diagonal hoppings. These can be thought of as arising from magnetic impurities residing halfway through the perpendicular $\left(t_{y}\right)$ hoppings and arranged antiferromagnetically.

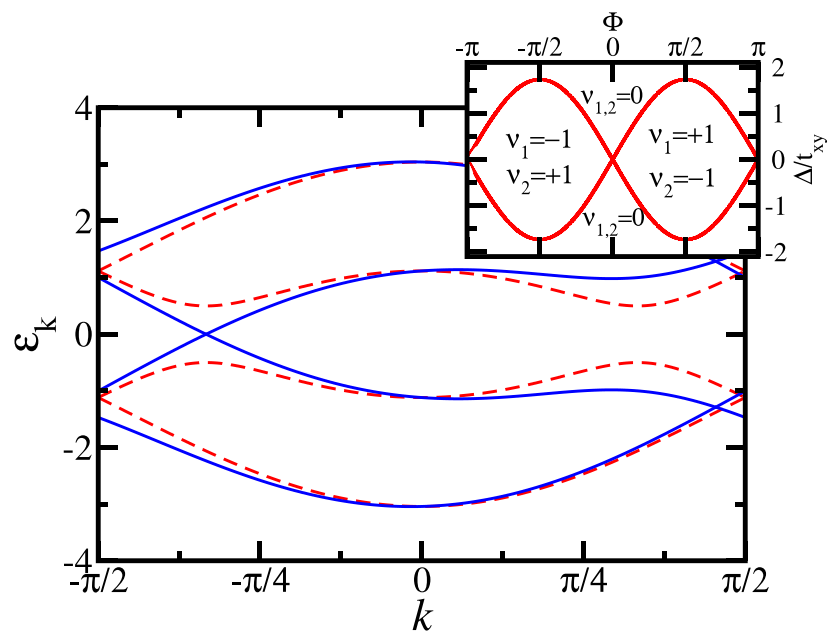

Figure 2. Band structure within the reduced Brillouin zone (RBZ). The red dashed lines indicate the band structure for a system with $t_{x}=1, t_{y}=1, t_{x y}=0, \Delta=0.5$. At half-filling this system is gapped. For $t_{y}=0$ the gaps would be at the edge of the RBZ $\left(k= \pm \frac{\pi}{2}\right)$.

Finite $t_{y}$ causes the gaps to move towards the origin (the gap is at $\left.k^{ \pm}= \pm \operatorname{acos}\left(\frac{t_{y}}{2 t_{x}}\right)\right)$. The blue solid lines indicate gap closure when $t_{x y}$ is made finite $\left(t_{x y} \sin \left(k^{ \pm}\right) \sin (\Phi)=\Delta,\left(\Phi=\frac{\pi}{2}\right)\right)$. The inset shows the phase diagram (where gap closure occurs) for $t_{x}=1, t_{y}=1$. The lines separate two insulating phases. The red(black) lines indicates gap closure occurring at $k^{+}\left(k^{-}\right)$.

To characterize the symmetry, let us consider the case $\Phi=\pi / 2$. PHS is achieved by $c_{k} \rightarrow c_{k}^{\dagger}$ and $c_{k}^{\dagger} \rightarrow c_{k}$ which reverses the signs of all matrix elements. PHS can also be realized by the operator $C=\mathrm{i}\left(I_{2} \otimes \tau_{y}\right)$, which squares to minus one. If $\Delta=0$ TRS is realized by $T=\left(I_{2} \otimes \tau_{x}\right) \kappa$, which squares to plus one, and the system is in the $C I$ symmetry class. Finite $\Delta$ breaks TRS, and the symmetry class of the model becomes $C$. The operator $R=\left(I_{2} \otimes \tau_{x}\right)$ can also act as the reflection of the different legs of the ladder. This operator anti-commutes with the PHS operator, but commutes with the TRS.

For a complete topological characterization, however, it is expedient to start with the Hamiltonian after applying the similarity transformation in equation (3). Considering the case $\Phi=\pi / 2$ for simplicity results in

$$
h(\pi / 2,0)=U^{-1} H(\pi / 2,0) U=h_{x} \otimes \tau_{x}+h_{z} \otimes \tau_{z},
$$

where $h_{x}$ is a $2 \times 2$ diagonal matrix with elements $-2 t_{x} \cos (k) \pm t_{y}$ and so is $h_{z}$ with $\Delta \mp 2 t_{x y} \sin (k)$. Hence, we have two decoupled subsystems whose Hamiltonian is similar to the model analyzed by Jackiw and Rebbi [18], where zero-mode edge states were already demonstrated. If one of the $2 \times 2$ models is Fourier transformed back to real space, the result is a Creutz model with a potential $\Delta$ on all the sites forming one leg of the ladder, and $-\Delta$ on the other. The symmetry characterization for $\Delta=0$ is BDI $[19,20]$, for finite $\Delta$ the TRS and PHS are broken, but CS is maintained, leading to AIII. Both of these have a $\mathbb{Z}$ topological index.

It is instructive to compare at this point to the spin-dependent 'doubling' situation in the Kane-Mele model. The KaneMele model can be constructed in two steps. One first takes two Haldane models, one for each spin, and couples them via a Rashba term. In the Haldane model TRS is broken, but in the combined system it is restored, and at TRS invariant $k$-points a degeneracy is guaranteed by Kramers theorem. Our model can be constructed by taking two extended Creutz models, which are not PHS invariant individually, but their combination is PHS-1. The Creutz models in our case are arrived at after transforming our original Hamiltonian, meaning that they are defined in terms of quasi-particles, rather than real particles on the lattice, hence the analog of 'doubling' is in terms of linear combinations of orbitals (the transformation in equation (3) combines sites in a unit cell with either both $\Delta$ or both $-\Delta$ ). There is no Kramers theorem, but a degeneracy can be produced by tuning the reflection and TRS breaking terms. The gap closure does not have to occur at TRS invariant points.

Extending the work of Ryu et al [3] we construct the topological index. The ground state projector can be written as

$$
P(k)=\frac{1}{2}\left[I_{4}-\bar{Q}(k)\right]=\frac{1}{2}\left[I_{4}-\mathbf{h}(k) \otimes \tau\right] .
$$

The matrix $\bar{Q}(k)$ can be brought into off-diagonal form $(Q(k))$ by applying the transformation

$$
\frac{1}{\sqrt{2}}\left[I_{2} \otimes\left(\begin{array}{cc}
1 & 1 \\
\mathrm{i} & -\mathrm{i}
\end{array}\right)\right],
$$

and switching the order of multiplication for the direct product we arrive at the $Q(k)$ matrix

$$
Q(k)=\left(\begin{array}{cc}
0 & q(k) \\
q(k)^{*} & 0
\end{array}\right),
$$

where $q(k)$ is a diagonal $2 \times 2$ matrix with elements $-h_{z}(k)-\mathrm{i} h_{x}(k)$. The winding number [3] is given by

$$
\nu=\frac{\mathrm{i}}{2 \pi} \int_{-\pi}^{\pi} \operatorname{Tr}\left[q^{-1}(k) \partial_{k} q(k)\right] \mathrm{d} k=\nu_{1}+\nu_{2},
$$

where

$$
\nu_{j}=\frac{\mathrm{i}}{2 \pi} \int_{-\pi}^{\pi}\left[q_{j}^{-1}(k) \partial_{k} q_{j}(k)\right] \mathrm{d} k
$$



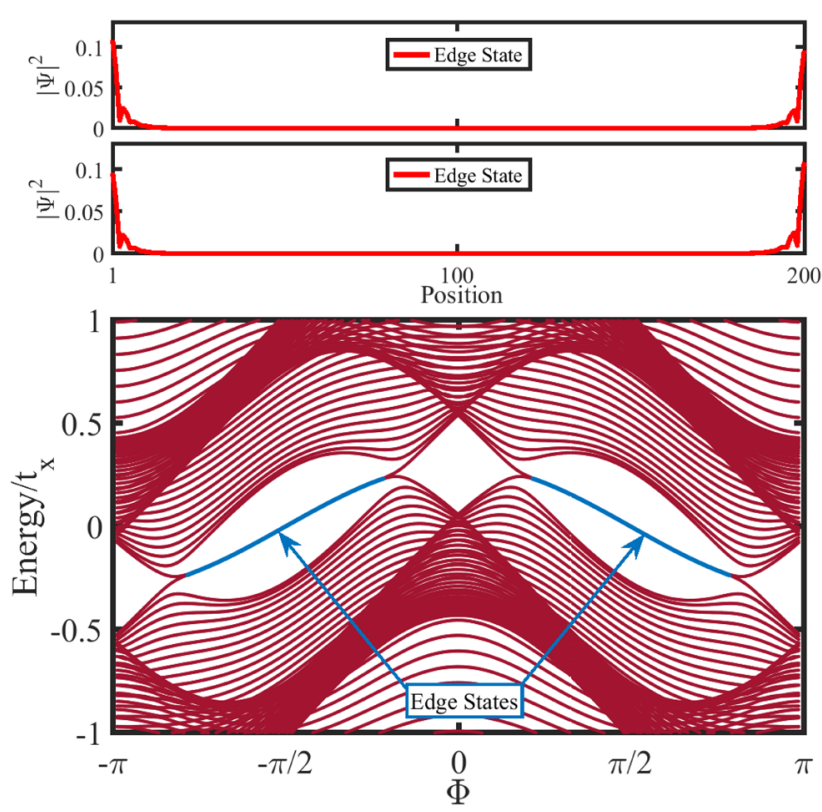

Figure 3. Lower panel: band structure of a system of size 100 sites with open boundary conditions. In this calculation $t_{x}=1, t_{y}=1$, and $t_{x y}=0.3$. Inside the lobes (see inset of figure 2) edge states arise. Upper two panel: squared modulus of the wavefunction for the two edge states averaged over the two legs of the ladder, for $\Phi=\pi / 2$ for a system of 200 sites.

with

$$
\begin{aligned}
& q_{1}(k)=\Delta-2 t_{x y} \sin (k)+\mathrm{i} t_{y}+2 \mathrm{i} t_{x} \cos (k) \\
& q_{2}(k)=\Delta+2 t_{x y} \sin (k)-\mathrm{i} t_{y}+2 \mathrm{i} t_{x} \cos (k) .
\end{aligned}
$$

The winding numbers can be written as contour integrals,

$$
\nu_{j}=\frac{(-1)^{j}}{2 \pi \mathrm{i}} \oint \frac{\mathrm{d} \mathbf{z}}{\mathbf{z}-\mathbf{z}_{j}}, j=1,2
$$

where the integral is around the ellipse defined by the curve

$$
\mathbf{z}=t_{x} \cos (k)+\mathrm{i} t_{x y} \sin (k),
$$

with $-\pi \leqslant k<\pi$, and

$$
\begin{aligned}
& \mathbf{z}_{1}=\frac{-t_{y}}{2}+\mathrm{i} \frac{\Delta}{2} \\
& \mathbf{z}_{2}=\frac{t_{y}}{2}+\mathrm{i} \frac{\Delta}{2} .
\end{aligned}
$$

Both winding numbers will be zero, if the points $\mathbf{z}_{1}$ and $\mathbf{z}_{2}$ fall outside the curve defined by equation (12). Since the curve is symmetric with respect to the imaginary axis, the two points will be either both inside the curve or outside. When the points are inside, $-\nu_{1}=1=\nu_{2}$. The topological index can be called the mirror winding number, the one-dimensional analog of the mirror Chern number $[9,10]$. The conditions for the points to fall on the curve correspond to the gap closure conditions derived above.

Figure 3 shows the band structure of the system under open boundary conditions. The lower panel shows the band structure when $\Phi$ is scanned between $-\pi, \pi$ for $t_{x}=t_{y}=1$, and $t_{x y}=0.3$. Inside the lobes of the phase diagram two edge states are found (indicated in blue color in the figure). The
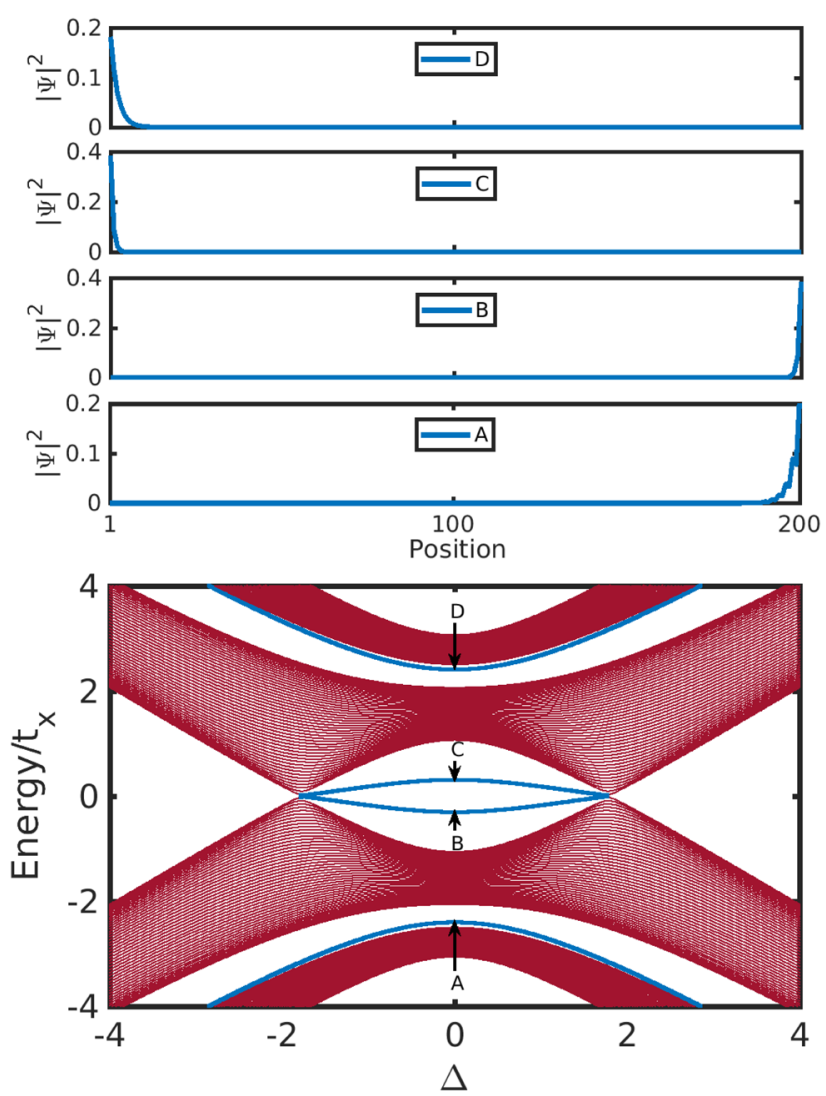

Figure 4. Band structure of system with finite $\Gamma=0.5$ and edge states (upper four panels). The other parameters are $t_{x}=t_{y}=t_{x y}=1, \Phi=\pi / 2$, and $\Phi_{B}=0$. The variable $\Delta$ is scanned. Localized edge states are found at quarter, half, and three-quarter fillings.

upper panels of the figure show the squared modulus of these edge states. Each one is localized near the ends of the ladder.

We have also considered the Hall response of the system, to a magnetic field perpendicular to the plane of the ladder. The Středa formula $[21,22]$ for the Hall conductance reads

$$
\sigma_{H}=\left.e c \frac{\Delta n}{\Delta \Phi_{B}}\right|_{\mu} .
$$

We applied a magnetic field by threading a flux $\Phi_{B}$ on the bonds with hopping parameter $t_{x}$ in opposite directions on each legs of the ladder (as indicated in figure 1). We first calculate the chemical potential for half-filling for $\Phi_{B}=0$. Subsequently we calculated the number of states below the chemical potential at $\Phi_{B}=0$ when the flux is changed by a flux quantum per unit cell. In the topologically non-trivial phase changing the flux in either direction leads to a decrease in the number of states below the chemical potential, by two particles. This happens for both positive and negative flux. In the topologically trivial phase there is no change in the number of particles when a flux is threaded.

We now investigate the model with $\Phi=0$, and a flux $\Phi_{B}=\pi / 2$, corresponding to a magnetic field perpendicular to the ladder. The behavior we find is very similar to what we found for the finite $\Phi$ case. The PHS operator in this case is $C=\left[\mathrm{i} \sigma_{y} \otimes \tau_{x}\right]$, again squaring to minus one $\left(\sigma_{x}, \sigma_{y}, \sigma_{z}\right.$ 
denote the Pauli matrices acting in the 'left' subspace). The TRS is $T=\left[\sigma_{x} \otimes I_{2}\right] K$, which squares to one, i.e. the system falls in the $C I$ symmetry class. The operator $\left[\sigma_{x} \otimes I_{2}\right]$ is also an inversion operator, although, unlike in the previous case, it inverts halfway along a chosen bond, horizontal in figure 1. For the case $\Delta=0$ we obtain a Hamiltonian similar to that in equation (2),

$$
\begin{aligned}
H(0, \pi / 2)=\sum_{k} & {\left[\sigma_{x} \otimes\left(\begin{array}{cc}
-2 t_{x y} \cos (k) & -t_{y} \\
-t_{y} & -2 t_{x y} \cos (k)
\end{array}\right)\right.} \\
& \left.+\sigma_{z} \otimes\left(\begin{array}{cc}
0 & -2 t_{x} \sin (k) \\
-2 t_{x} \sin (k) & 0
\end{array}\right)\right] .
\end{aligned}
$$

Here we can apply the similarity transformation via

$$
V=\frac{1}{\sqrt{2}}\left[I_{2} \otimes\left(\begin{array}{cc}
1 & 1 \\
1 & -1
\end{array}\right)\right],
$$

and arrive at

$h(0, \pi / 2)=V^{-1} H(0, \pi / 2) V=\sigma_{x} \otimes g_{x}+\sigma_{z} \otimes g_{z}$,

with $g_{x}$ and $g_{z}$ being $2 \times 2$ diagonal matrices with elements $-2 t_{x y} \cos (k) \mp t_{y}$ and $\mp 2 t_{x} \sin (k)$, respectively. The two $2 \times 2$ models which form the $4 \times 4$ model are two Creutz models $[14,15]$ with band structures displaced with respect to each other by $\pi$. The transformation in equation (16) combines sites of the same ladder leg within a unit cell, therefore the Creutz models in this case are defined in terms of quasi-particles of this kind. The gap closures occur at $k=0$ and $\pi$ when $t_{y}= \pm 2 t_{x y}$. We can again construct the mirror winding number scheme used above. For the topological state the curve

$$
\mathbf{z}=t_{x y} \cos (k)+\mathrm{i} t_{x} \sin (k)
$$

will include the points $\pm t_{y} / 2$, with winding numbers of opposite signs. If these points are outside the curve, both winding numbers will be zero. It is well-known that the Creutz model is topological and exhibits edge states [14].

For finite values of $\Delta$ the topological state survives, since the state is adiabatically connected to the $\Delta=0$ state. The phase diagram can be determined from the fact that the gap closure has to occur at $k=0$ (a single point in the reduced Brillouin zone). Setting $k$ to zero in the Hamiltonian we can diagonalize the resulting matrix, and obtain the gap closure condition,

$$
\Delta= \pm \sqrt{\left(4 t_{x y}^{2}-t_{y}^{2}\right)\left(1-\left(\frac{t_{x}}{t_{x y}} \cos \left(\Phi_{B}\right)\right)^{2}\right)} .
$$

At last, for the case $\Phi=\pi / 2$ we consider adding a term of the form $\Gamma\left[\sigma_{z} \otimes \tau_{z}\right]$, which still preserves PHS, but mixes the two Creutz-like subsystems (in this sense an analog of the Rashba term in the Kane-Mele model). The $q$-matrix in this case becomes

$$
q(k)=h_{x}+\mathrm{i} h_{z}+\left(\begin{array}{cc}
0 & \Gamma \\
\Gamma & 0
\end{array}\right) .
$$

Although the derivation is more cumbersome than in the previous case, due to the off-diagonal elements of the $q$-matrix, the winding number falls into the same two pieces as in equations (8)-(13), the gap closure conditions for the two contributions are the same as before.

In figure 4 the band structure is shown for a system with $\Gamma=0.5, t_{x}=t_{y}=t_{x y}=1, \Phi=\pi / 2, \Phi_{B}=0$ scanned over the variable $\Delta$. The four midgap states, two at half filling, one at quarter and three-quarter fillings are evaluated at $\Delta=0$. The upper panels show that these midgap states are indeed localized edge states. The state at half-filling are non-degenerate, unlike for zero $\Gamma$. Note that the states form 'particle-hole' pairs: for a given negative energy state, there is a positive energy state which is localized on the other edge.

\section{Conclusion}

We have constructed a ladder model (one-dimensional) which falls in the $C$ or $C I$ symmetry classes and exhibits topological behavior, as a result of two types of reflection symmetries present. The models can be shown to consist of two submodels. In this respect, the situation is similar to the 'Haldane squared' model (the Kane-Mele model without the Rashba coupling) which exhibits a spin-resolved quantum spin-Hall effect. The two submodels on their own exhibit nonzero winding numbers, which are of opposite sign in the topologically nontrivial phase. While a condensed matter realization of all the interesting parameter ranges seems a challenge, however, ladder models can be realized as ultracold atoms in optical lattices [23], even the analogs of topologically nontrivial models $[24,25]$.

\section{Acknowledgment}

BH gratefully acknowledges support from the Simons Center for Geometry and Physics, Stony Brook University at which some of the research for this work was performed.

\section{ORCID iDs}

B Hetényi (ㅇ https://orcid.org/0000-0002-3680-1147 M Yahyavi (10 https://orcid.org/0000-0003-0062-203X

\section{References}

[1] Altland A and Zirnbauer M R 1997 Phys. Rev. B 551142

[2] Schnyder A P, Ryu S, Furusaki A and Ludwig A W W 2008 Phys. Rev. B 78195125

[3] Ryu S, Schnyder A P, Furusaki A and Ludwig A W W 2010 New J. Phys. 12065010

[4] Kitaev A Yu 2009 AIP Conf. Proc. 113422

[5] Kane C L and Mele E J 2005 Phys. Rev. Lett. 95226801

[6] Kane C L and Mele E J 2005 Phys. Rev. Lett. 95146802

[7] Chiu C-K, Yao H and Ryu S 2013 Phys. Rev. B 88075142

[8] Chiu C-K, Teo J C Y and Schnyder A P 2016 Rev. Mod. Phys. 88035005

[9] Teo J C Y, Fu L and Kane C L 2008 Phys. Rev. B 78045426

[10] Lau A, van den Brink J and Ortix C 2016 Phys. Rev. B 94165164

[11] Volovik G E 1997 JETP Lett. 66522

[12] Schnyder A P, Ryu S and Ludwig A W W 2009 Phys. Rev. Lett. 102196804

[13] Deng D-L, Wang S-T and Duan L-M 2014 Phys. Rev. B 89075126 
[14] Creutz M and Horváth I 1994 Phys. Rev. D 502297

[15] Creutz M 1999 Phys. Rev. Lett. 832636

[16] Haldane F D M 1988 Phys. Rev. Lett. 612015

[17] Li L, Xu Z and Chen S 2014 Phys. Rev. B 89085111

[18] Jackiw R and Rebbi C 1976 Phys. Rev. D 133398

[19] Sticlet D, Seabra L, Pollmann F and Cayssol J 2014 Phys. Rev. B 89115430
[20] Sticlet D, Dóra B and Cayssol J 2013 Phys. Rev. B 88205401

[21] Středa P 1982 J. Phys. C: Solid State Phys. 15 L717

[22] Widom A 1982 Phys. Lett. A 90474

[23] Bloch I 2008 Nature 4531016

[24] Sun K, Liu W V, Hemmerich A and Das Sarma S 2012 Nat. Phys. 867

[25] Mancini M et al 2015 Science 3491510 